Elsevier Editorial System(tm) for Colloids and Surfaces A: Physicochemical and Engineering Aspects

Manuscript Draft

Manuscript Number: COLSUA-D-12-00956R1

Title: Statistical properties and morphology of a 2D gel network at the air/water interface.

Article Type: Special Issue : Bubble\&Drop Interfaces

Keywords: 2D Gel, Langmuir monolayer, gold nanoparticles, percolating network, scaling law

Corresponding Author: Prof. Luigi Cristofolini, Ph.D. Corresponding Author's Institution: University of Parma, Italy

First Author: Davide Orsi

Order of Authors: Davide Orsi; Alessandro Vezzani; Raffaella Burioni; Andrea Pucci; Giacomo Ruggeri; Luigi Cristofolini

Abstract: We report here a detailed characterization of the steps leading to the formation of a bidimensional gel at the air water interface. The 2D geometry is peculiar in that it allows a continuous and controlled variation of the density of the system over a wide range. The evolution of the statistical properties of the structured network, as a function of the externally controlled density, is related to the corresponding evolution of the mechanical properties. We identified three steps in the gel formation: a first step occurs in the incubation time, in which the individual nm-sized gold nanoparticle aggregate to form quasi 1D structures of typical length of a few microns. The second step occurs in the first stages of the compression, with the growth of the branched structure, finally yielding to the onset of the infinite percolative cluster, which is related to the building of the mechanical elastic modulus. The final step identifies with the homogenization and regularization of the distribution of holes sizes, which relates to the further increase of the elastic modulus upon compression. We speculate that this study should help in the formulation of gels with desired characteristics, such as increased mechanical strength, or increased mobility . 


\section{Dear Editor,}

please find enclosed the revised version of our manuscript, which takes into account all the observations by the referee, whom we thank for the kind appreciation words "...which makes the manuscript very interesting and original. It can be published after minor revision...."

Coming to the detailed points raised:

Point 1: We expanded the "Discussion" section, to include a comparison with the results obtained by the groups of Radke and of Noskov on interfacial gels formed by protein solutions, and 4 relevant references have been added.

Point 2: we still do not understand completely the dynamical mechanism giving rise to the complex observed pattern. As the critical exponents we measure are different from the ones predicted for 2dimensional percolation, a diffusion mechanism or a steric effect are expected to play an important role. Nevertheless, all these mechanisms predict a universal behavior of the scaling exponents, which typically depend on dimensionality. We therefore expect that the measured value should be characteristic of the $2 \mathrm{~d}$ case and they should change in 3 dimensional systems. We included this point in the Discussion, with a reference to a paper by Hidalgo et al.

Finally, about point 3: in paragraph 3.1 we clarified the experimental reasons for which the initial times are not accessed by the present study, and explained why the data in figures 1 and 3 cannot refer to the early times of aggregation.

With this, we think we have fulfilled all the indications of the referee, and we think the manuscript should be publishable.

Sincerely

Luigi Cristofolini 

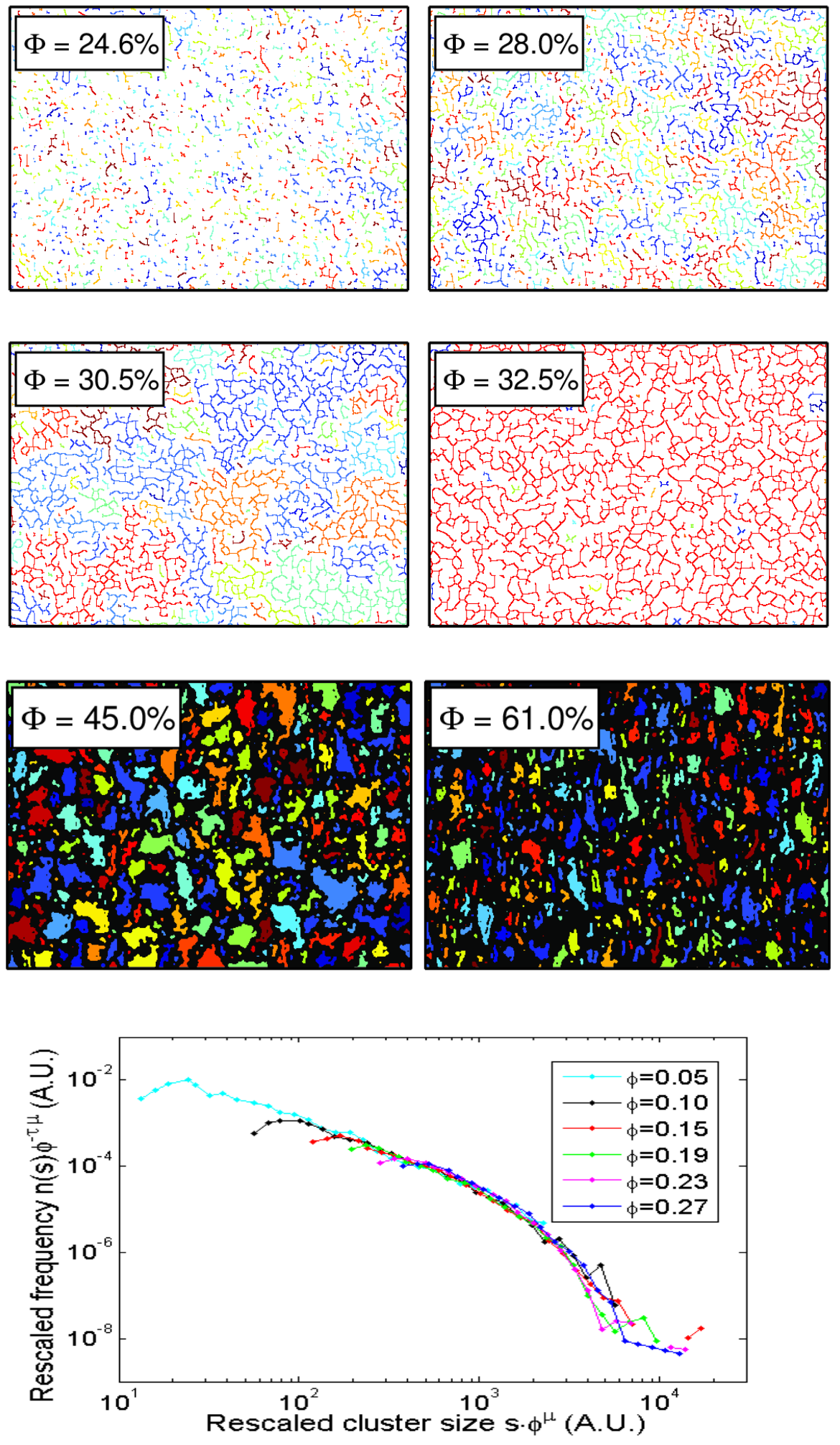


\section{Highlights:}

- A 2D gel system is formed by a Langmuir film of gold nanoparticles

- Its microscopic structure is characterized

- Three steps are identified in the formation of the gel:

- Incubation and formation of quasi1D micrometric aggregates

- Formation of the infinite percolative cluster

- Homogenization of the resulting hole structure

- The last two steps are described by a sophisticated theoretical scaling approach

- This is expected to be beneficial to the design of novel gels with enhanced functionality (e.g. stronger from the mechanical point of view, or on the contrary, characterized by enhanced mobility). 


\title{
Statistical properties and morphology of a 2D gel network at the air/water interface.
}

\author{
Davide Orsi ${ }^{1}$, Alessandro Vezzani ${ }^{1,3}$, Raffaella Burioni ${ }^{1,4}$, Andrea Pucci $^{2}$, Giacomo Ruggeri ${ }^{2}$, Luigi \\ Cristofolini $^{1 *}$ \\ ${ }^{1}$ Department of Physics and Earth Sciences, University of Parma, \\ Viale Usberti 7A, 43124 Parma, Italy \\ ${ }^{2}$ Department of Chemistry and Industrial Chemistry, University of Pisa, \\ Via Risorgimento 35, 56126 Pisa, Italy \\ ${ }^{3}$ Centro S3, CNR-Istituto di Nanoscienze, Via Campi 213A, 41125 Modena Italy \\ ${ }^{4}$ INFN, Gruppo Collegato di Parma, viale G.P. Usberti 7/A, 43124 Parma, Italy
}

\author{
*corresponding author: \\ prof. Luigi Cristofolini \\ Department of Physics and Earth Sciences, University of Parma, \\ Viale Usberti 7A, 43124 Parma, ITALY \\ e-mail: $\quad$ Luigi.Cristofolini@unipr.it \\ Tel: $\quad+390521905276$ \\ Fax: $\quad+390521905223$
}

Keywords: 2D Gel, Langmuir monolayer, gold nanoparticles, percolating network, scaling law

\begin{abstract}
We report here a detailed characterization of the steps leading to the formation of a bidimensional gel at the air water interface. The 2D geometry is peculiar in that it allows a continuous and controlled variation of the density of the system over a wide range. The evolution of the statistical properties of the structured network, as a function of the externally controlled density, is related to the corresponding evolution of the mechanical properties. We identified three steps in the gel formation: a first step occurs in the incubation time, in which the individual $\mathrm{nm}$-sized gold nanoparticle aggregate to form quasi 1D structures of typical length of a few microns. The second step occurs in the first stages of the compression, with the growth of the branched structure, finally yielding to the onset of the infinite percolative cluster, which is related to the building of the mechanical elastic modulus. The final step identifies with the homogenization and regularization of the distribution of holes sizes, which relates to the further increase of the elastic modulus upon compression. We speculate that this study should help in the formulation of gels with desired characteristics, such as increased mechanical strength, or increased mobility.
\end{abstract}

\section{1 - Introduction}

Systems with a high surface-to-volume ratio are common in everyday life, like in foods (beer foam, with its stability and consistency, or the softness and fragrance of levitated dough), cosmetics 
(shaving foams, and pastes) and pharmaceuticals (plasters for the controlled release of drugs) just to mention a few. In all these systems, a particular role is played by gel networks, in which a given substance (a protein, or inert colloidal particles) forms a branched structure. This structure, even if it is not filling the space, determines the mechanical properties of the system, while the empty spaces contain a different phase (liquid or gaseous), which in turn may convey molecules imparting new desired functionalities. The statistics of the connections between different gel branches mark the difference between different type of gels. Also the dynamics of these systems, and in particular the possibility for a given molecule or particle to travel along long distances, is a consequence of the topological and statistical properties of the network; as a matter of fact, the distribution of connections and holes can strongly influence transport and can lead to anomalous diffusive properties, ranging from subdiffusive [1] to superdiffusive dynamics [2,3,4]. In this framework, architectures formed by inorganic nanoparticles (NP) are particularly interesting as they can be exploited in an increasing number of technological applications, including biosensing, therapeutics and diagnostics.

The basic scientific knowledge in this area feeds into many applications in chemical, pharmaceutical and food industries, as well as in designing nano-devices such as sensors, assays, photonics and bio-fuel cells. These devices in turn are deployed in the areas of energy, health and environmental protection.

NPs can also be used as "additives" to improve the performance of existing materials (as thermal conductivity, mechanical stability or energy transfer) [5], or to impart new functions to them (e.g. magnetic NPs have been investigated for drug delivery) [6,7]. From the fundamental point of view, NPs bridge the length scales between molecular surfactants and micron-sized particles. The former usually are in dynamical equilibrium with a bulk reservoir, whereas the latter are irreversibly attached to the surface as first shown by Pieransky [8]. NPs are somehow in between the two extremes and can be in dynamical equilibrium between surface and subphase, depending on their coating and on the surface pressure.

Recently a new type of colloidal 2D gels formed in mixed Langmuir monolayers of stearic acid and octadecylamine was reported on a surface of gold hydrosol. The adsorption of gold nanoparticles on the mixed mono-layer led to an increase of interactions between oppositely charged surfactants giving a "soap" of mixed fatty salt. [9] The consequent shrinking and rearrangement of the monolayer resulted in aggregation of nanoparticles into colloidal 2D "soap"-gels. A similar structure was characterized by a combination of techniques accessing the spontaneous fluctuations together with the mesoscopic scale mechanical properties, as probed by Interfacial Shear Rheology [10-13]

\section{2 - Material and methods}

Dodecanthiol-stabilized gold nanoparticles (GNPs) have been synthesized via the two-phase Brust method [14]: nanoparticles have been prepared by dissolving $0.400 \mathrm{~g}(1.02 \mathrm{mmol})$ of hydrogen tetrachloro-aurate(III) trihydrate in $30 \mathrm{~mL}$ of deionized water. The solution has been then shaken in a separatory funnel with $80 \mathrm{~mL}$ of toluene solution containing $2.00 \mathrm{~g}$ (3.56 mmol) of tetra-noctylammonium bromide (TOAB). The toluene phase has been then recovered and combined with $0.018 \mathrm{~mL}(0.015 \mathrm{~g}, 0.07 \mathrm{mmol})$ of dodecyl mercaptane. A freshly prepared aqueous solution of sodium borohydride $(25 \mathrm{~mL}, 0.386 \mathrm{~g})$ has been slowly added under vigorous stirring. After further stirring for $3 \mathrm{~h}$, the organic phase has been separated, concentrated to $10 \mathrm{~mL}$ and mixed with $70 \mathrm{~mL}$ of ethanol. The mixture has been cooled overnight at $-20^{\circ} \mathrm{C}$ and the dark precipitate then recovered by filtration. The crude product has been then purified by soxhlet extraction with acetone as cleansing solvent to remove all the unbound free thiol and residual TOAB impurities, thus making Au NPs fully soluble in organic solvents. The average diameter of DT-AuNPs has been estimated to be $8 \mathrm{~nm}$ by DLS analysis with a $6 \mathrm{~nm}$ diameter gold nucleus (TEM analysis) [15]. 
Uniform Langmuir monolayers have been prepared on a Langmuir trough with two moving barriers (maximum surface $20 \times 5 \mathrm{~mm}^{2}$ ), equipped with a circular quartz window. The trough is placed on the moving table of a Nikon-Eclipse inverted microscope.

It must be noted that the dodecanethiol coating provides a hydrophobic interaction, which stabilizes the particles when dispersed in an organic solvent (e.g. in toluene), so that the bulk suspensions are stable for months after preparation. On the contrary, when dispersed at the air/water interface, the same organic coating seems to provide an attractive interaction between the particles, which then aggregate at low concentration, thus forming the branched 2D network structure that is investigated in this work.

To form the Langmuir monolayer, as originally suggested by Chen and co-workers [16] an hexanechloroform 9:1 suspension of gold nanoparticles (concentration $1 \mathrm{mg} / \mathrm{ml}$ ) was spread on the water surface using a $50 \mu \mathrm{l}$ Hamilton syringe, while keeping the tip of the needle in contact with the water surface. The spreading of a single syringe of solution required roughly 5 minutes, with frequent changes of the position of the needle's tip on the water surface. This was followed by a waiting time of 10 minutes for solvent evaporation. These waiting times have to be carefully abided in order to obtain reproducible results. This procedure was repeated to reach the total amount of $200 \mu \mathrm{L}$ of solution spread. Measurements were performed at room temperature $\left(25^{\circ} \mathrm{C}\right)$. A Wilhelmy balance was used to measure the surface pressure of the air/water interface: in previous works, we demonstrated that the measurement of this parameter can be used to track the variations of the surface concentration of the film [10]. The Wilhelmy plate was kept perpendicular to the moving barriers, and thus parallel to the direction of the uniaxial compression of the film, during all the measurements.

Typical isotherms were recorded analogue to that already reported in [10]. The trough is placed on the moving table of a Nikon-Eclipse inverted microscope, equipped with extra-long working distance (ELWD) Nikon objectives. Since the optical system of this microscope is infinitycorrected, we were able to extend the optical path and to place the microscope objective close to the quartz window of the Langmuir trough: in this way, we were able to acquire images of the film at the air/water interface. All the images reported in this work were acquired using a 20X ELWD (working distance: $8 \mathrm{~mm}$ ) objective.

Images were acquired with a peltier-cooled Andor Clara CCD camera, capable of acquiring $1392 \times 1024$ 16bit pixels at 10 frames per seconds, with $5 \mathrm{~ms}$ exposure time. The camera was interfaced through the Micromanager software [17]. Digital images were analyzed using the Matlab Image Processing Toolbox by subtracting a background image, and then binarized when necessary by applying an adaptive threshold algorithm.

\section{3 - Results}

\subsection{First stages in the formation of the film}

We emphasize that the empiric recipe for the formation of a good quality film of nanoparticles [16] requires that after the spreading of the suspension of the gold nanoparticles at the air/water interface, the surface is left at rest for an incubation time, and several addition and waiting cycles need to be performed. Then the film can be formed by slow compression: given the importance of this early stage protocol we decided to investigate it directly. The aggregation process described in the work is driven by quasi-stationary, slow compression of the sample obtained by closing the barriers of the Langmuir trough. However, the initial aggregation of the nanoscopic gold colloids, taking place at low concentration after -and during- the spreading procedure, cannot be directly followed by our experimental setup: the aggregates are not distinguishable from the background until the network is starting to form, at surface concentrations significantly higher. At this point, in fact, the string-like aggregates have reached a size of a few micron, sufficiently high to be clearly distinguishable in the transmission geometry of the experiment.

At this point, a movie ( 1 frame per second) was recorded during compression up to a surface 
pressure of $5 \mathrm{mN} / \mathrm{m}$, corresponding to a surface concentration of roughly $\Phi=30 \%$. Six frames from this movie are reported in the Figure 1, representative of the film organization at the different values of concentration indicated in the figure: a network-like structure is starting to appear roughly at $\Phi=25 \%$, yielding to a closed structure gel as the concentration is further increased.

In order to investigate on the nature of the film, we calculated the radial dependence of the mass $\mathrm{M}(\mathrm{R})$, which in a regular lattice should follow a power law of $\mathrm{R}$, growing linearly for a simple 1D structure, quadratically for a homogeneous 2D structure, and should follow an intermediate power law for a fractal. The experimental results for the lower concentrations are shown in the left panel of figure 2, in which we observe a quasi-linear growth for small length scale, indicative of a 1D structure, up to a crossover length located at 4-5 $\mu \mathrm{m}$. Above this crossover length the growth of $\mathrm{M}(\mathrm{R})$ becomes quadratic, indicative of a 2D structure.

In order to investigate the average properties of the system, in the right panel of the same figure 2 we report several pseudo structure factors $\underline{S}(\mathrm{Q})$, calculated by radial averaging the Fourier transform of the binarized images. The position of the first peak in $\underline{S}(\mathrm{Q})$ corresponds, via a shape dependent factor, to the size of the objects. As the concentration is increased, the typical size of such objects increases slightly, as indicated by the gradual shift of the peak towards smaller Q values as the concentration is increased. The overall size variation is of the order of $15-20 \%$. This behavior was observed for all the low concentrations investigated (i.e. below 30\%) and can be interpreted by describing the progressive film formation as the aggregation of objects of linear shape, which have been previously formed during the incubation time. 

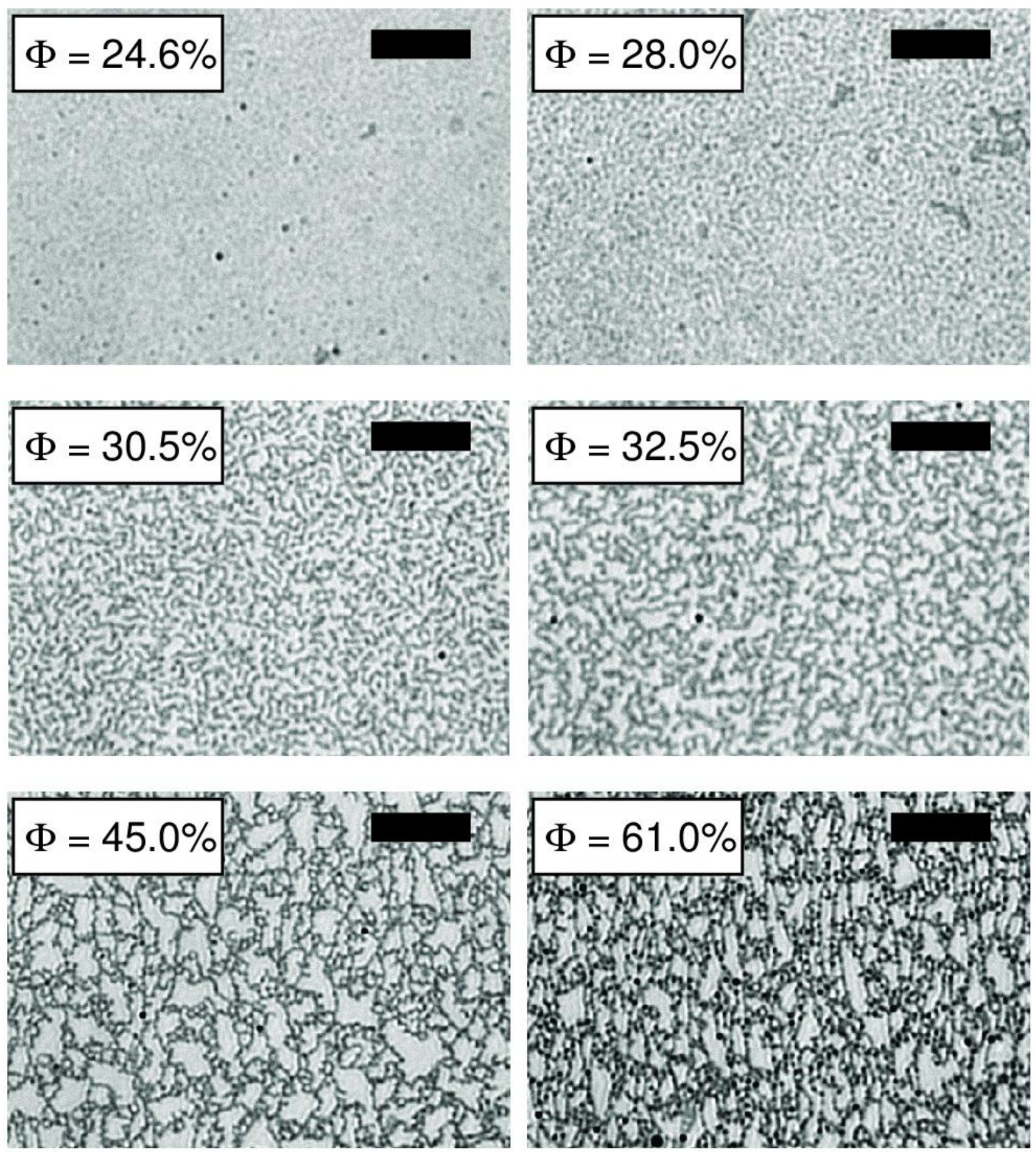

Figure 1: formation of the 2D gel network as the surface concentration Фof GNPs is increased. The images, recorded using the inverted microscope described in section 2, show the evolution of the film structure as $\Phi$ is increased, as indicate in each image. At the highest concentration a network is formed. The black strip (50 um long) provides a length scale reference. 

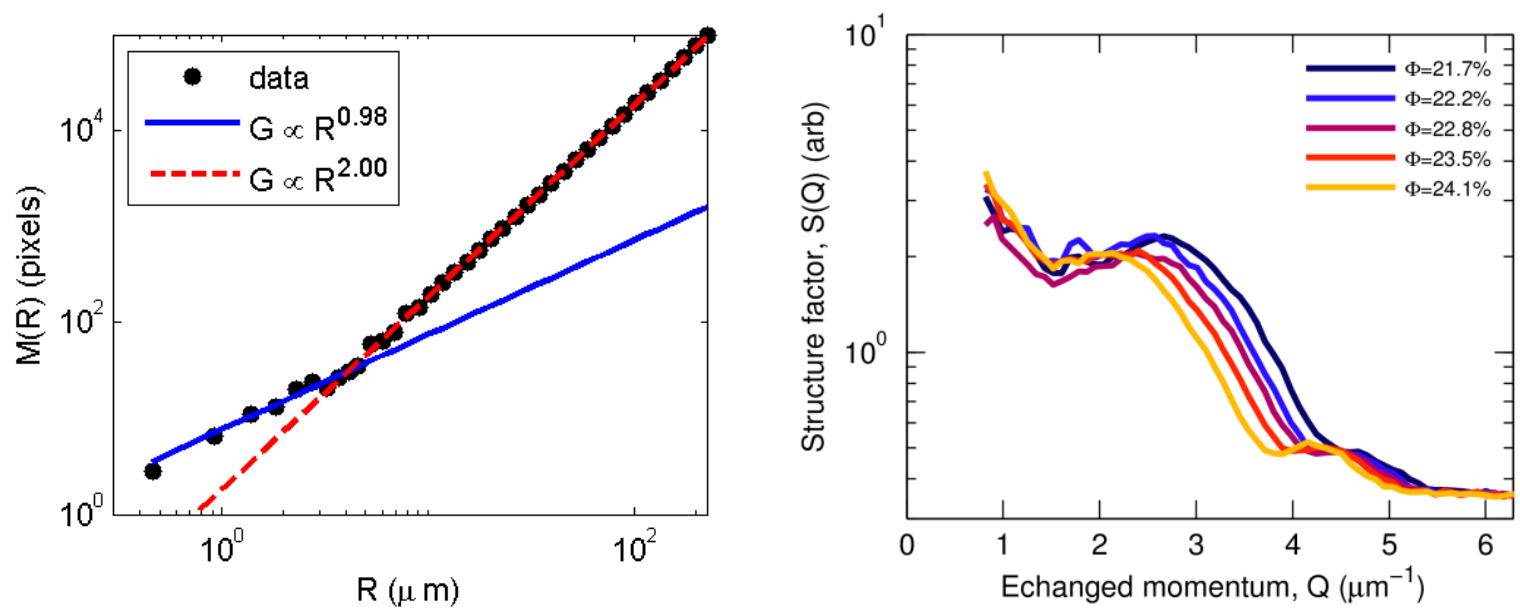

Figure 2: Small and quasi $1 D$ objects organize in a large scale homogeneous 2D film. Left:

radial averaged density of mass $M(R)$ calculated for $\Phi=23.5 \%$. Note the crossover (around 4-5 $\mu m$ ) between a linear and a quadratic growth the of the mass. Right: logarithm of the pseudo - structure factor $\underline{\mathrm{S}}(Q)$ (see text for details) as a function of the momentum, for different surface concentrations, indicated in the legend. Note that the position of the first peak shifts toward low values of $Q$ as concentration is raised.

\subsection{Towards a percolating network}
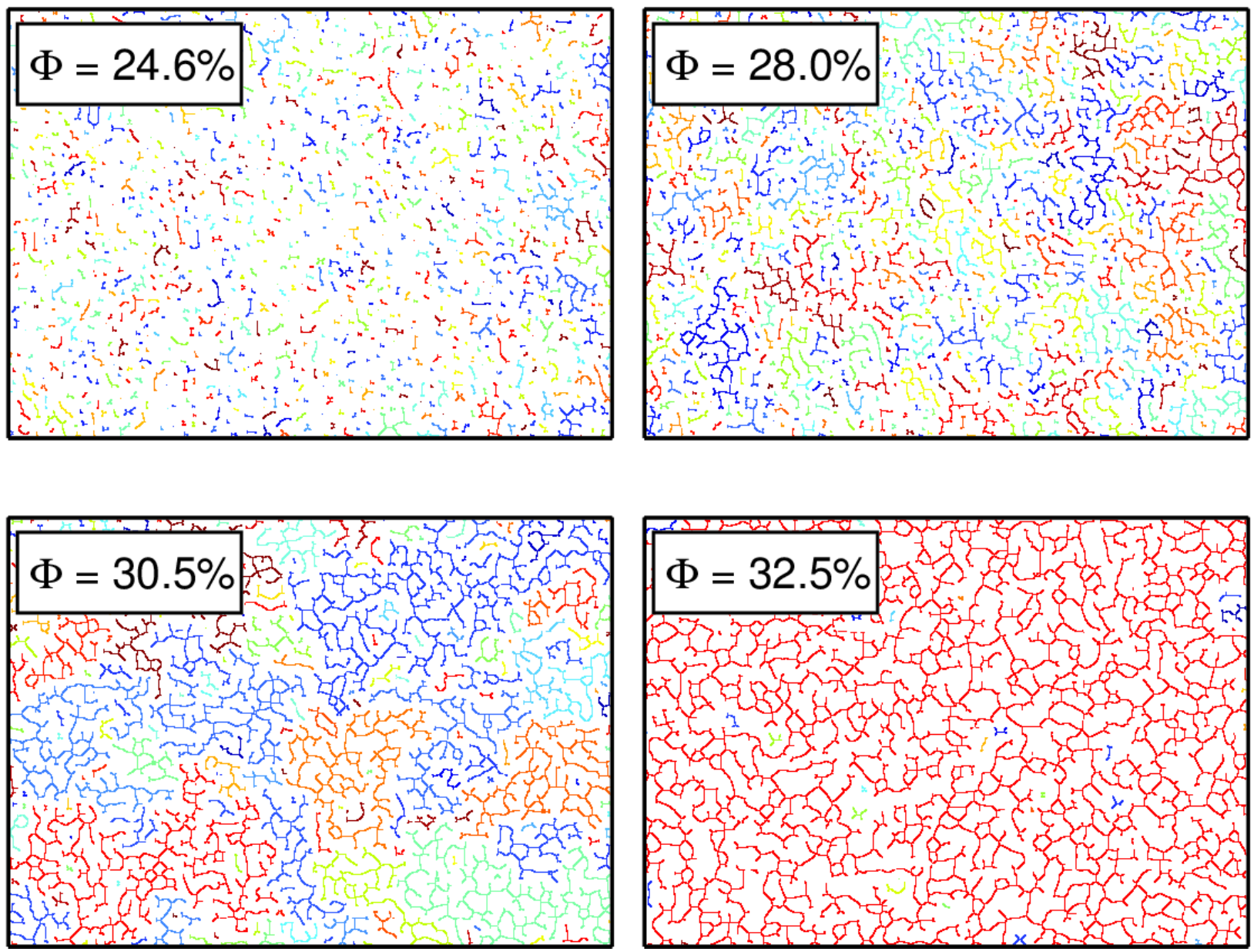

Figure 3: formation of the 2D network of GNPs. The panels report the binary skeletonization of the images reported in figure 1, showing that the network has percolated at $\Phi>32 \%$. Different colors indicate distinct clusters of the network. Scale bar are the same as in figure 1. 
As compression proceeds further, the aggregation process of the quasi 1D objects yields to a branched structure whose connectivity grows to finally form a percolating cluster extending over the whole observed area. In figure 3 we report the binary skeletonized structure, obtained at selected concentrations from the binarization of the pictures of figure 1 . The different clusters are indicated by different colors, and the onset of the percolative cluster is indicated by the appearance of the single (red) cluster in the bottom right panel.

In order to investigate in detail the formation of the percolative cluster we report in figure 4 the size $\left(s_{\max }\right)$ of the maximum cluster found in each image as a function of $\Phi$. Cluster size was evaluated by counting the pixels connected to each cluster, after image binarization and skeletonization. We note that this size can be very large in the presence of branched shapes.

The continuous line in figure 4 represents the fit to the theoretical model for second order phase transitions predicting a critical divergence described by the power law $s_{\max } \propto\left(\Phi-\Phi_{C}\right)^{\mu}$. Experimental data are best reproduced by assuming $\Phi_{C}=31.9(2)$ and $\mu=1.7(3)$. Note that above the critical divergence, the maximum cluster size obtainable corresponds to the filling of the whole image, which appears as the plateau in figure.

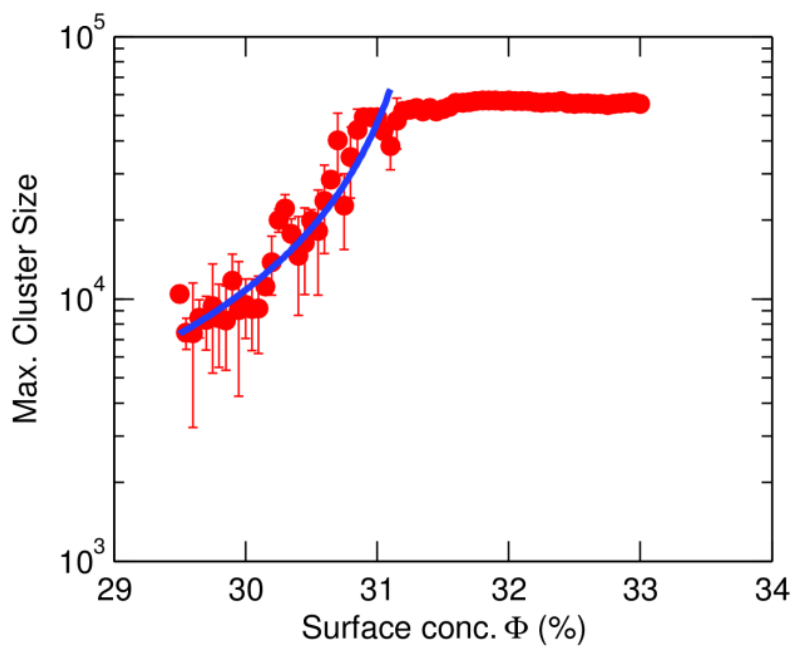

Figure 4: A percolative cluster is formed above $\Phi_{C}=31.9 \%$. Points: concentration dependence of the size of the largest cluster, on a logarithmic scale. Its growth is compatible with the predicted divergence represented by the continuous line. Above the critical divergence, the maximum size keeps the constant value allowed by the finite size of the images.

The distribution of cluster sizes s, representative of the different regimes of the gel, is reported in figure 5, left panel, as a function of concentration $\Phi$ and of cluster size on a logarithmic scale. Vertical cuts of the same surface are reported in the right panel of the same figure in which the frequency of cluster size is reported for different fixed values of $\Phi$ on a double logarithmic scale. It is evident that a power law scaling is obeyed in proximity of the critical regime, implying the lack of a single "typical size" for the clusters. On the contrary, at lower concentrations, the decay of the distribution is sharper, yielding a distribution with a "typical size" above which the frequency drops. 

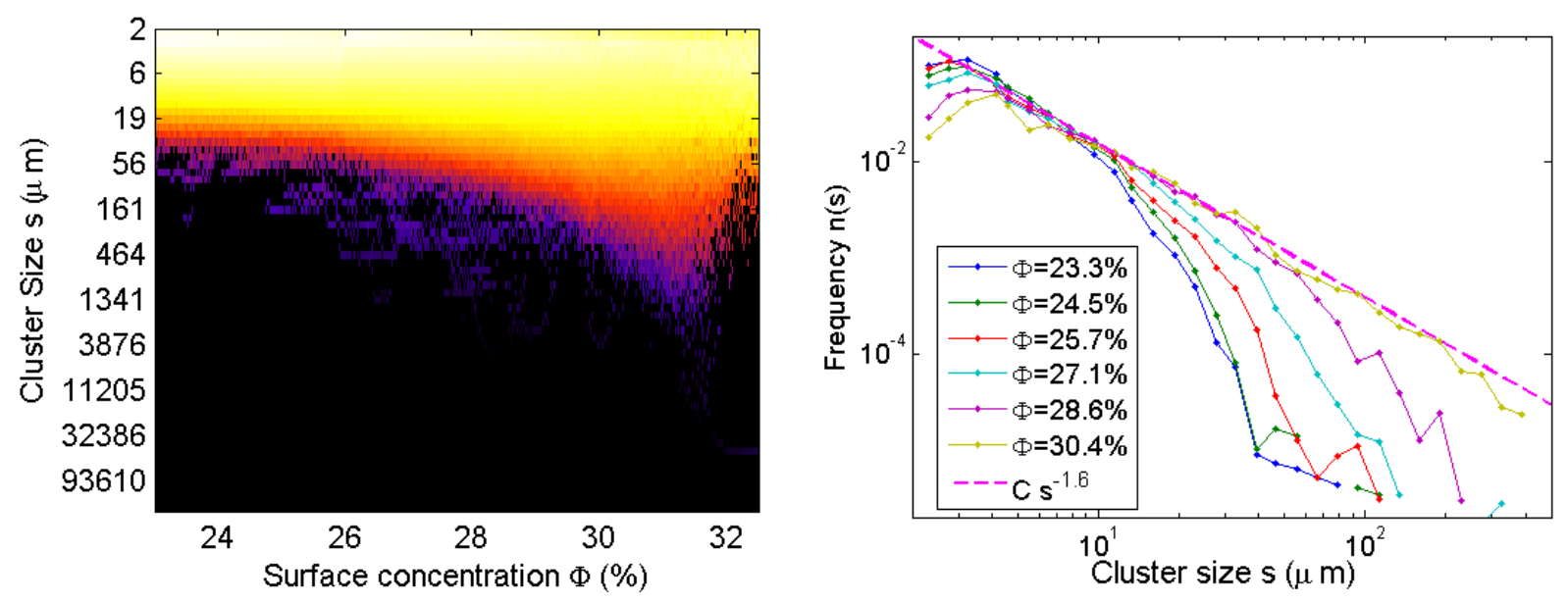

Figure 5: the distributions of cluster sizes decay with different laws: a power law at the criticality, and an exponential decay elsewhere. Left) Frequency of appearance of cluster sizes, as a function of the concentration $\Phi$. Right) Clusters sizes distributions. The straight line corresponds to the critical regime power law decay with exponent-1.6(2). Far from the critical point a faster decay is evident.

\subsection{The formed gel: a Lévy-distributed heterogeneous structures}
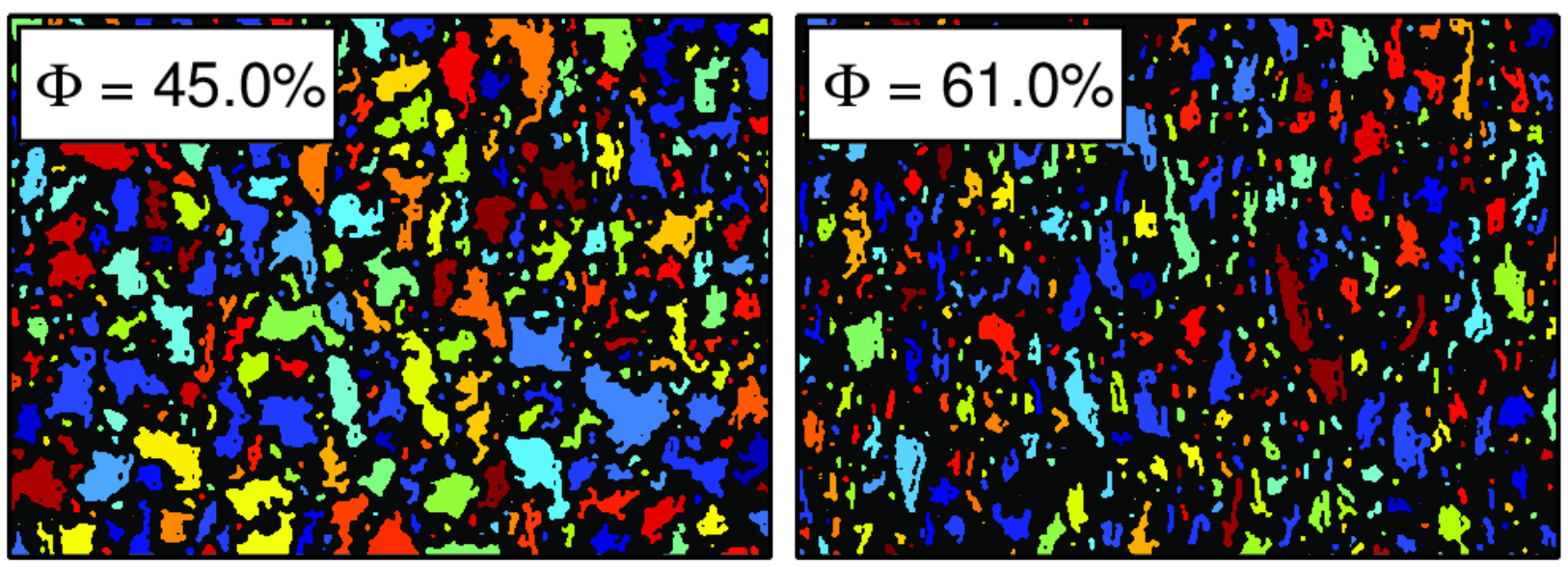

Figure 6: as the surface concentration of the film is increased the population of large holes is reduced. Binarized images, distinct holes are labeled with different colors. Scale bar are the same as in figure 1.

Above the critical point the network percolates the whole observed surface, and the film properties start to be governed by the holes size distribution. In figure 6 we report the same pictures of the last two panels of figure 1, corresponding to a high concentration film, converted to binary images by applying a threshold. Then, distinct holes are labeled with different colors.

In the left panel of figure 7 we report the corresponding distribution of the values of areas of the 
holes (A) for two selected concentrations $\Phi$. The continuous lines correspond to fits to the Lévy distribution $n(A)$ decaying as a power law $A^{-\alpha}$. In the right panel, we display the evolution of the exponent $\alpha$ which grows on increasing surface coverage $\Phi$. The fact that $\alpha$ lays between 1 and 2 implies that a mean value can be identified for the holes sizes, but at the same time they exhibit an infinite variance. The growth of $\alpha$ at high concentrations corresponds then to a more homogeneous structure.
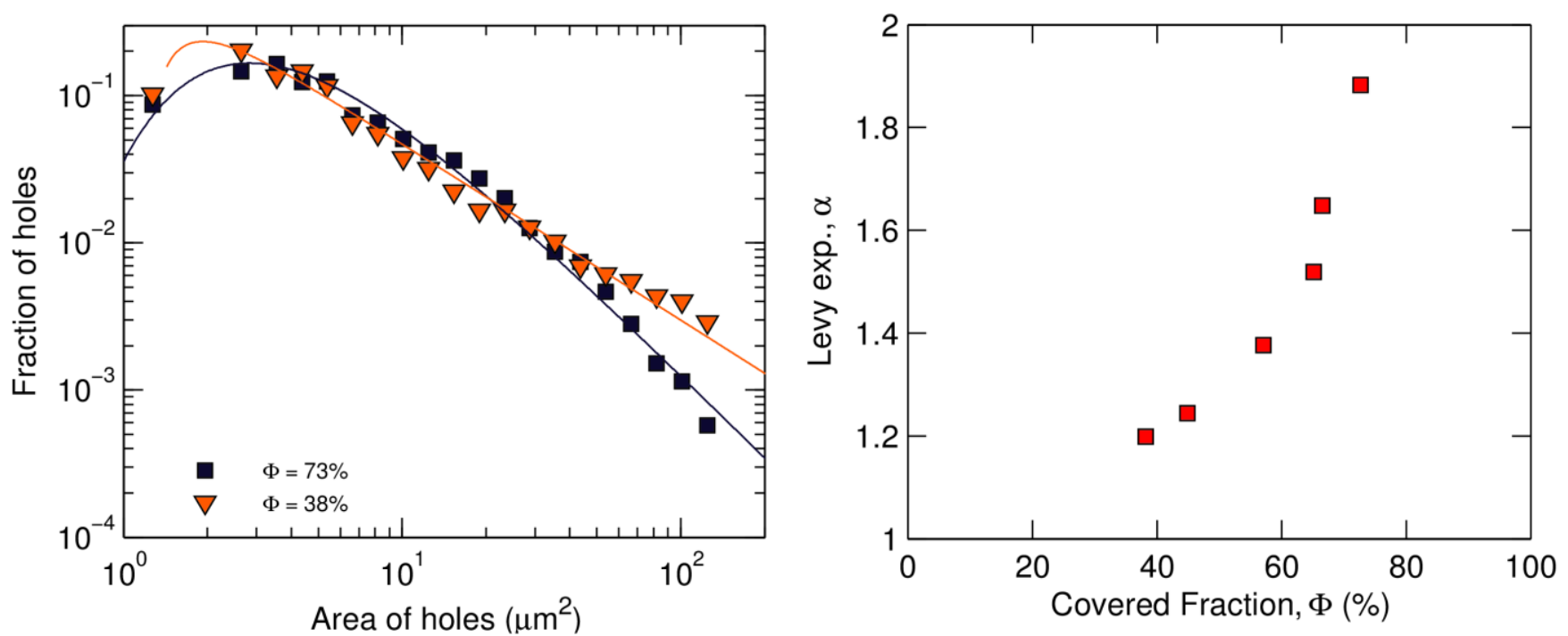

Figure 7: The distribution of holes sizes follows a Lévy curve. The power law exponent of its tail grows with concentration. Left) distribution of holes sizes, measured at two different values of $\Phi$ and reported in logarithmic scale. The continuous lines are fits to the Lévy distribution described in the text. Right) Exponent $\alpha$ of the algebraic tail of the Lévy distribution, reported as a function of the concentration.

\section{4 - Discussion}

Albeit the formation of interfacial gels is widely reported- and their mechanical properties thoroughly characterized - for solutions of proteins pure or mixed with surfactants [18-20] also in the presence of denaturizing agents [21], or at the fluid/fluid interface [22] much less is known for the purely inorganic gels formed by nanoparticles confined at an interface. Here we have shown that the structural properties of the GNP's film present interesting geometrical features, which can be tuned by a controlled and almost continuous variation of the density. At low concentrations, under the critical density, the network formed by the particles is characterized by elongated quasi $1 \mathrm{D}$ cluster, whose length grows slowly with the density. The typical pattern observed in figures 3-5 suggests a direct comparison with 2D percolation. For a more detailed discussion of the transition, we focus on the clusters sizes distribution, as described in figure 5. According to standard analysis of the percolation phase transition [22], the data suggest, for the density of clusters of size s, n(s), a scaling behavior of the form:

$$
n(s)=s^{-\tau} f\left(s \cdot \varphi^{\mu}\right)
$$


where $\varphi=\left(\Phi_{\mathrm{c}}-\Phi\right) / \Phi_{\mathrm{c}}$ is the reduced density, with $\Phi$ and $\Phi_{\mathrm{c}}$ representing the particle density and the critical particle density respectively and $f(x)$ a scaling function. Eq. (1) indicates that at criticality (i.e. $\varphi=0$ ) the clusters density decays as $\mathrm{s}^{-\tau}$. Moreover, for $\varphi \neq 0$ the density $\mathrm{n}(\mathrm{s})$ features the same power law behavior in the region $s<<\varphi^{-\mu}$. Indeed in this situation, one has that $s^{\cdot} \varphi^{\mu} \approx 0$ and $\mathrm{f}\left(\mathrm{s} \cdot \varphi^{\mu}\right) \approx \mathrm{f}(0)$, while for larger clusters the fast decaying function $\mathrm{f}(\mathrm{x})$ dominates the behavior of $\mathrm{n}(\mathrm{s})$, which then rapidly vanishes, typically in an exponential way. It is then clear that the qualitative behavior described by formula (1) fits well the experimental data presented in figure 5.

From the data collected in figure 4, we can evaluate the scaling exponents characterizing Eq. (1). The value of the exponent $\tau$ can be obtained directly from the data by fitting the power law behavior of $\mathrm{n}(\mathrm{s})$ when $\varphi \approx 0$. As evidenced from the continuous line in figure 5, $\tau$ can be estimated as $\tau$ $=1.6 \pm 0.2$.

The value of $\mu$ can be obtained taking into account that $\varphi^{-\mu}$ represents the typical size at which $\mathrm{n}(\mathrm{s})$ becomes vanishingly small, and therefore we expect that the largest cluster of the system $s_{\max }$ grows when $\varphi$ goes to zero as $\varphi^{-\mu}$. The data fitting presented in figure 4 provides an estimate for $\Phi_{\mathrm{c}}=$ $31.9 \pm 0.2$ and for the exponent $\mu=1.8 \pm 0.2$. Let us check that the data in figure 5 agree with the above values of the critical exponents. Formula (1) can be reshaped as follows:

$$
\mathrm{n}(\mathrm{s})=\varphi^{\tau \cdot \mu} \mathrm{g}\left(\mathrm{s} \cdot \varphi^{\mu}\right)
$$

where the new scaling function is $g(x)=x^{-\tau} f\left(x^{\mu}\right)$. In the first panel of figure 8 we plot $\varphi^{-\tau} n(s)$ as a function of $s^{\cdot} \cdot \varphi^{\mu}$, evidencing that data pertaining to different particle densities collapse on a single function $\mathrm{g}(\cdot)$. This behavior confirms that the cluster distribution exhibits the typical features of a second order phase transition, which can be studied within a scaling approach. The value we have obtained for the scaling exponents i.e. $\mu=1.8 \pm 0.2$ and $\tau=1.6 \pm 0.2$ are different from the analytically known exponents characterizing percolation in 2dimension $\mu_{\mathrm{c}}=91 / 36 \approx 2.5$ and $\tau_{\mathrm{c}}=187 / 91 \approx 2.05$. Panel 2 of figure 6 evidences that if one applies the scaling approach using the $2 \mathrm{D}$ percolation exponents the data collapse fails.

The values of the critical exponents are expected to be universal and independent of the local details of the system, such as the nature of the short-range interactions, the size of the elementary clusters or the characteristic time scales of local evolution. They are expected to depend only on the dimensionality of the space and on the symmetries of the models.

The experimental setup suggests that the dynamics leading to the formation of the clusters could be inherently different from the standard 2D percolation for two reasons. First, in percolation clusters are static and they are formed by adding at each step a new particle in a randomly chosen site. As here clusters are created dynamically, since the particles diffuse in time, the cluster formation should resemble in some way to the typical features of DLA (diffusion limited aggregation) [24]. In DLA, a highly ramified cluster is generated around an original seed by clusters that diffuse and stick to the main cluster when they touch one of its branch. However, at difference with DLA, here the particle density is not fixed and the cluster is not generated around a single seed. Second, in the experimental set up one of the spatial symmetry of the 2D system is broken. Indeed the particle density is increased by reducing the surface of the system according to a preferential direction i.e. performing a uniaxial compression of the film. In this way the up-down and left right direction are no more equivalent and a spatial symmetry turns out to be broken.

For the same dynamical and geometrical reasons the short length shape of the elementary clusters in this systems turns out to be elongated providing a further difference with respect standard percolation. 

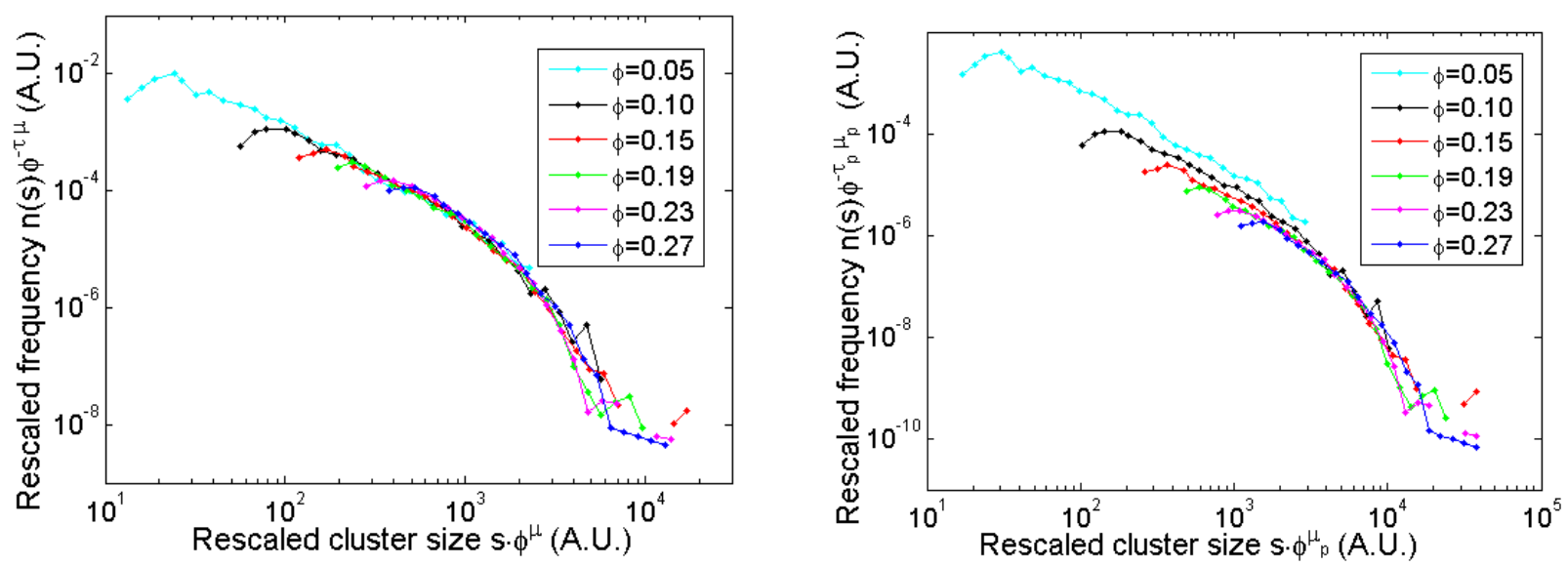

Figure: 8 Rescaled particle density according to formula (2) Left) the scaling exponents are fixed to be $\mu=1.8 \pm 0.2$ and $\tau=1.6 \pm 0.2$ and a good data collapse is evidenced Right) the scaling exponents are fixed according to standard $2 D$ percolation and the data collapse fails.

Finally, It is important to note that the elastic mechanical response of the gel originates from the presence of a macroscopic cluster, which identifies with the microscopically observed percolative cluster. In our previous report [11], we found that at low surface concentrations the mechanical modulus of the GNP gel film was too small to be measured (due to a corresponding low Boussinesq number), while above a critical concentration of about $27 \%$ the film displayed a measurable elastic response, which remarkably follows a scaling law in the reduced concentration. The present findings provide a direct structural explanation of this phenomenon.

Furthermore, the growth of the mechanical modulus with the surface concentration goes together with the development of a homogeneous structure, as identified by the increase in the Lévy exponent $\alpha$.

It should be noted however that in the present study we find that the critical concentration for the onset of the percolative infinite cluster is $\Phi c=31.9 \%$, while the mechanical data could be fitted by assuming the smaller value $\Phi c=27 \%$. This suggests that the mechanical properties start to be affected much earlier than the onset of the percolative cluster. The threshold value in that case could be identified when the clusters reach a size large enough that their drag starts to be significantly larger than that provided by the subphase. It should be noted however that this is only slightly larger than the accuracy in our surface concentration measurements which is typically of the order of 1$2 \%$, being obtained by accurate cross validating of the data from different measurements: in situ null-ellipsometry, SEM imaging of deposited monolayers, and surface pressure versus area (П/A) measurements.

\section{5 - Conclusions}

The present study provides a structural explanation of our previous findings on the mechanical properties of 2D gels formed by gold nanoparticles at the air water interface. In particular we can identify three steps in the 2D gel formation: a first step occurs in the incubation time, in which the individual nm-sized gold nanoparticle aggregate to form quasi 1D structures of typical length of a few microns. The second step occurs during first stages of the compression, with the increase of the size of the branched structure, finally yielding to the onset of the infinite percolative cluster, which is related to the building of the mechanical elastic modulus. The final step identifies with the homogenization and regularization of the distribution of holes sizes, which relates to the further 
increase of the elastic modulus upon compression.

We also demonstrated the onset of a non-gaussian distribution of features, as probed by the divergence of the distribution momentum. The transition from the low density to the high density phase features the typical scaling behavior observed in second order phase transitions. However, the measured scaling exponents do not fall in the universality class of 2D percolation, suggesting a more complex dynamics in the network formation.

Indeed, the leading mechanism giving rise to the structures we observe is not clear yet. We expect an interplay between diffusion limited aggregation (DLA) and percolation driven effects. In both cases, it is well known that the critical exponents feature an universal behavior and they typically depend on the dimensionality of the system [25]. Therefore the scaling exponent we measure should be characteristic of the $2 \mathrm{D}$ case.

A by-product of this study could be the suggestion of how different preparation routes and interparticle interactions should help in the production of 2D gels characterized by increased strength (more branched) or on the contrary enhanced mobility (larger holes). Parameters that could be varied, in view of this result, are the interparticle interaction (as driven by chemical surface functionalization), the surface diffusivity (which could be controlled also by forming mixed monolayers with low molecular weight surfactants) and the holes size distribution (which can be controlled by appropriate compression cycles).

\section{Acknowledgements}

We would like to acknowledge the "Fondazione Cassa di Risparmio di Parma" for funding the Project "Microscopia in fluorescenza in sistemi molecolari complessi di interesse biomedico" which made possible the acquisition of the inverted microscope used in the present study.

\section{Bibliography}

[1] J.P. Bouchaud and A. Georges, Phys. Rep. 195127 (1990)

[2] P. Levitz, Europhys. Lett. 79 593-598 (1997)

[3] M. Palombo, A. Gabrielli, S. De Santis, C. Cametti, G. Ruocco, and S. Capuani, J. Chem. Phys. 135, 034504 (2011)

[4] P. Buonsante, R. Burioni, and A. Vezzani, Phys. Rev. E 84, 021105 (2011)

[5] A.C. Balazs, T. Emrick, T.P. Russell, Science, 314, 1107-1110 (2006)

[6] X. Zhao, J. Kim, C.A. Cezar, N. Huebsch, K. Lee, K. Bouhadir, D.J. Mooney, Proceedings of the National Academy of Sciences of the United States of America 108, 67-72 (2011)

[7] S. Erokhina, L. Cristofolini, T. Berzina, V. Erokhin, M.P. Fontana, Journal of Magnetism and Magnetic Materials 272-276, 1353-1354 (2004)

[8] P. Pieranski, Physical Review Letters 45, 569-572 (1980)

[9] D. I Babenko, A. Ezhov, D. S Turygin, V. Ivanov, V. V. Arslanov, and M. Kalinina, Langmuir 28, 125-33 (2012) 
[10] D. Orsi, L. Cristofolini, G. Baldi, and A. Madsen, Physical Review Letters 108, 105701 (2012).

[11] L. Cristofolini, M.P. Fontana Philosophical Magazine B84, 1537, (2004).

[12] D. Orsi, G. Baldi, P. Cicuta, and L. Cristofolini, Journal of Colloids and Surfaces A, 413,71-77 (2012)

[13] D. Orsi L. Cristofolini, M.P. Fontana , J. of Non-Crystalline Solids 357, 580-586 (2011)

[14] M. Brust, M. Walker, D. Bethell, D. J. Schiffrin and R. Whyman, J. Chem. Soc., Chem. Commun., 801-802 (1994)

[15] M. Dionisio, F. Maffei, E. Rampazzo, L. Prodi, A. Pucci, G. Ruggeri, E. Dalcanale Chem. Commun., 47, 6596-6598 (2011)

[16] X. Y. Chen, J. R. Li, and L. Jiang, Nanotechnology 11, 108 (2000)

[17] A. Edelstein, N. Amodaj, K. Hoover, R. Vale, and N. Stuurman, Current Protocols in Molecular Biology 14.20.1-14.20.17, (2010)

[18] B.A. Noskov and M. M. Tikhonov Colloid J. 74, 248-253 (2012)

[19] B. A. Noskov, O. Y. Milyeaeva, S. Lin, G. Loglio, R. Miller Coll. Surf. A 413, 84-91 (2012)

[20] A. Maestro, C. Kotsmar, A. Javadi, R. Miller, G. Loglio J. Phys. Chem. B, 116, 4898-4907 (2012)

[21] B. A. Noskov, O. Y. Milyeaeva, S. Lin, G. Loglio, R. Miller Langmuir 26,17225-17231 (2010)

[22] E.M. Freer, K.S. Yim, G.G. Fuller, C.J. Radke J. Phys. Chem. B, 108, 3835-3844 (2004)

[23] D. Stauffer and A. Aharony, "Introduction to percolation theory", Taylor and Francis (2003)

[24] T.A.Witten, L. A. Sanders Phys. Rev. Lett. 47, 1400-1403 (1981)

[25] A. E. Gonzalez, F. Martinez-Lopez, A. Moncho-Jorda, R. Hidalgo-Alvarez, Physica A 314, 235-245 (2002) 